\section{Cáncer en Chile y en el mundo: una mirada actual y su futuro escenario epidemiológico}

\author{
SOLANGE PARRA-SOTO ${ }^{1,2, a}$, FANNY PETERMANN-ROCHA ${ }^{1,2, a}$, \\ MARÍA ADELA MARTÍNEZ-SANGUINETTI ${ }^{3, \mathrm{~b}}$, \\ ANA MARÍA LEIVA-ORDEÑEZ ${ }^{4, c}$, \\ CLAUDIA TRONCOSO-PANTOJA ${ }^{5, \mathrm{a}}$, NATALIA ULLOA ${ }^{6,7, \mathrm{~b}, \mathrm{e}}$, \\ XIMENA DIAZ-MARTÍNEZ ${ }^{8, \mathrm{~d}, \mathrm{e}}$, CARLOS CELIS-MORALES $^{1,2,9,10, \mathrm{~d}, \mathrm{e}}$
}

\section{Cancer in Chile and worldwide: an overview of the current and future epidemiological context}

Cancer is a chronic non-communicable disease associated with a high mortality burden. The prevalence of cancer is increasing rapidly worldwide. However, this scenario will be worse in low and middle-income countries such as Chile, where $70 \%$ of cancer deaths occur. The aim of this review was to assess the epidemiological scenario of cancer and its projection for the Chilean population. In Chile, 53,365 new cases of cancer were diagnosed in 2018, led by prostate, colorectal, breast, stomach, lung and gallbladder cancer. From 1986 to 2016, cancer increased by 109\%. When we reviewed mortality by sex, stomach and prostate cancer were responsible for more than $30 \%$ of cancer deaths among men. However, for women the first three places were occupied by breast, colorectal and lung cancer, as in the rest of the world. Considering that $40 \%$ of cancers are related to unhealthy lifestyles, working on the prevention of modifiable risk factors represents an opportunity for the creation of public health policies that allow changes at the environmental and individual level.

(Rev Med Chile 2020; 148: 1489-1495)

Key words: Epidemiology; Neoplasms; Mortality.

${ }^{1}$ Institute of Health and Wellbeing, University
of Glasgow. Glasgow, United Kingdom.
${ }^{2}$ Institute of Cardiovascular and Medical
Sciences, University of Glasgow. Glasgow,
United Kingdom.
${ }^{3}$ Instituto de Farmacia, Facultad de Ciencias,
Universidad Austral de Chile. Valdivia, Chile.
${ }^{4}$ Instituto de Anatomía, Histología y
Patología, Facultad de Medicina, Universidad
Austral de Chile. Valdivia, Chile.
${ }^{5}$ CIEDE-UCSC, Departamento de Salud
Pública, Facultad de Medicina, Universidad
Católica de la Santísima Concepción.
Concepción, Chile.
${ }^{6}$ Centro de Vida Saludable, Universidad de
Concepción. Concepción, Chile.
${ }^{7}$ Departamento de Bioquímica Clínica
e Inmunología, Facultad de Farmacia,
Universidad de Concepción. Concepción,
Chile.
${ }^{8}$ Departamento Ciencias de la Educación,
Universidad del Bío-Bío.Chillán, Chile.
${ }^{9}$ Centro de Investigación en Fisiología del
Ejercicio (CIFE), Universidad Mayor. Santiago,
Chile.
10Laboratorio de Rendimiento Humano,
Grupo de Estudio en Educación, Actividad
Física y Salud (GEEAFyS), Universidad
Católica del Maule. Talca, Chile.
anutricionista.
${ }^{b}$ Bioquímico.
Profesora de Biología y Química.
dProfesor Educación Física.
eDoctor en Ciencias.
Recibido el 6 de diciembre de 2019,
aceptado el 9 de julio de 2020.
Correspondencia a:
Carlos Celis-Morales
Institute of Health and Wellbeing.
University of Glasgow. Glasgow, United
Carlos.Celis@glasgow.ac.uk

'Institute of Health and Wellbeing, University Sciences, University of Glasgow. Glasgow, United Kingdom.

${ }^{3}$ Instituto de Farmacia, Facultad de Ciencias, Universidad Austral de Chile. Valdivia, Chile. Austral de Chile. Valdivia, Chile.

${ }^{5}$ CIEDE-UCSC, Departamento de Salud Pública, Facultad de Medicina, Universidad Católica de la Santísima Concepción. Concepción, Chile.

${ }^{6}$ Centro de Vida Saludable, Universidad de Concepción. Concepción, Chile.

${ }^{7}$ Departamento de Bioquímica Clínica e Inmunología, Facultad de Farmacia,

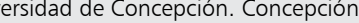
Universidad del Bío-Bío.Chillán, Chile. ${ }^{9}$ Centro de Investigación en Fisiología de (CIFE), Universidad Mayor. Santiago,

${ }^{10}$ Laboratorio de Rendimiento Humano Grupo de Estudio en Educación, Activida Física y Salud (GEEAFyS), Universidad Católica del Maule. Talca, Chile.

${ }^{a}$ Nutricionista.

Profesor Educación Física.

Recibido el 6 de diciembre de 2019

Institute of Health and Wellbeing Kingdom.

Carlos.Celis@glasgow.ac.uk
$\mathrm{D}$ urante los últimos 20 años, el cáncer se ha transformado en una las principales causas de mortalidad a nivel mundial. En el año 2018, se diagnosticaron un total de 18,1 millones de personas con cáncer y 9,6 millones murieron a causa de esta enfermedad ${ }^{1}$. Con las actuales tasas de crecimiento, cambios en los estilos de vida y envejecimiento de la población, se proyecta que para el 2040 habrá un incremento de $60 \%$ en el número de casos, llegando a 21,7 millones de nuevos diagnósticos de cáncer y 13,1 millones de muertes asociadas a esta enfermedad ${ }^{2}$.
Este escenario será incluso menos alentador en países de ingreso económico medio y bajo, donde habrá un incremento de $81 \%$ en el número personas con cáncer dentro de las próximas dos décadas ${ }^{3,4}$.

La población chilena no estará ajena a este incremento en el número de casos y muertes por cáncer. Durante el 2018 se diagnosticaron más de 53.000 casos de cáncer en Chile, según la última estadística de Global Cancer Observatory (Globocan $)^{5}$. Considerando este escenario, es de suma relevancia revisar las actualizaciones en la evi- 
dencia científica sobre cambios en la prevalencia y proyecciones de cánceres específicos en nuestra población. Conocer esta información permitirá tomar todas las medidas de control necesarias, desde las dirigidas a la prevención hasta el diagnóstico, tratamiento, gestión, cuidados paliativos y vigilancia. En este contexto, el objetivo de esta revisión narrativa de la literatura fue contextualizar la información disponible en la población chilena, en relación con el escenario epidemiológico del cáncer y su proyección a nivel nacional y mundial ${ }^{4}$.

\section{Materiales y Métodos}

La presente revisión narrativa de la literatura se realizó bajo la siguiente metodología. Se realizó la pregunta, ¿cuál es la situación actual del cáncer en el mundo y en Chile?, en base a ello se buscaron los últimos reportes mundiales y nacionales en las páginas webs oficiales de la Organización Mundial de la Salud (OMS), Globocan, Agencia Internacional para la Investigación en Cáncer (AIRC), Cancer Research UK y Ministerio de Salud de Chile. Se seleccionaron solo los reportes que responderían la pregunta y tuvieran las estadísticas más actuales a nivel mundial y para Chile.

\section{Escenario epidemiológico del cáncer}

El año 2018 se estimó que, a nivel mundial, aproximadamente 9,6 millones de personas
(56,4\% hombres y $43,6 \%$ mujeres) murieron por causa del cáncer, siendo los tipos de cánceres con mayor mortalidad: cáncer de pulmón, colorrectal, estómago, hígado, mama, esófago, páncreas, próstata, cérvico uterino y leucemia. Segun datos de AIRC se observa que por sexo, en los hombres el cáncer de pulmón fue la primera causa de muerte y en el caso de las mujeres el cáncer de mama, seguido por el cáncer de pulmón y colorrectal (Tabla 1$)^{6}$.

La última estadística de la AIRC, correspondiente al año 2018, señala que se diagnosticaron 18,1 millones de casos de cáncer, de los cuales $52,3 \%$ correspondían a hombres ${ }^{1}$. El cáncer de pulmón lidera la tabla de cánceres con mayor incidencia, seguido por el cáncer de mama, colorrectal, próstata, estómago, hígado, esófago, cérvico uterino, tiroides y vejiga. Al revisar el ranking de incidencia según sexo, los hombres presentan una tendencia similar a la del total de la población, siendo el cáncer de pulmón la primera causa seguido del cáncer de próstata. El cáncer de mama es el principal cáncer para el sexo femenino, seguido del cáncer colorrectal y pulmón ${ }^{7}$ (Tabla 2 ). La evidencia sugiere que 1 de cada 8 mujeres desarrollará cáncer de mama a nivel mundial ${ }^{8}$. La genética es responsable de tan solo 5 a $10 \%$ de todos los casos, el 90 a 95\% restante está vinculada a factores del medio ambiente y estilo de vida ${ }^{9}$.

En el caso de Chile, la mortalidad por cáncer ha aumentado en 109\% entre los años 1986 a

Tabla 1. Ranking de cánceres con mayor nivel de mortalidad a nivel mundial y según sexo, AIRC 2018

\begin{tabular}{|c|c|c|c|c|c|c|}
\hline \multirow[t]{2}{*}{ Ranking } & \multicolumn{2}{|c|}{ Total } & \multicolumn{2}{|c|}{ Hombres } & \multicolumn{2}{|c|}{ Mujeres } \\
\hline & $\begin{array}{l}\text { Tipo de } \\
\text { cáncer }\end{array}$ & $\begin{array}{c}\text { Muertes } \\
\text { n (\%) }\end{array}$ & $\begin{array}{l}\text { Tipo de } \\
\text { cáncer }\end{array}$ & $\begin{array}{c}\text { Muertes } \\
\text { n (\%) }\end{array}$ & $\begin{array}{l}\text { Tipo de } \\
\text { cáncer }\end{array}$ & $\begin{array}{c}\text { Muertes } \\
\text { n (\%) }\end{array}$ \\
\hline 1 & Pulmón & $1.761 .007(18,4 \%)$ & Pulmón & $1.184 .947(22,0 \%)$ & Mama & $626.679(15 \%)$ \\
\hline 2 & Colorrectal & $880.792(9,2 \%)$ & Hígado & $548.375(10,2 \%)$ & Pulmón & $576.060(13,8 \%)$ \\
\hline 3 & Estómago & $782.685(8,2 \%)$ & Estómago & $513.555(9,5 \%)$ & Colorrectal & $396.568 \quad(9,5 \%)$ \\
\hline 4 & Hígado & 781.631 (8,2\%) & Colorrectal & $484.224(9,0 \%)$ & Cérvico uterino & $311.365 \quad(7,5 \%)$ \\
\hline 5 & Mama & $626.679(6,6 \%)$ & Próstata & $358.989(6,7 \%)$ & Estómago & $269.130 \quad(6,5 \%)$ \\
\hline 6 & Esófago & $508.585(5,3 \%)$ & Esófago & $357.190(6,6 \%)$ & Hígado & $233.256 \quad(5,6 \%)$ \\
\hline 7 & Páncreas & $432.242(4,5 \%)$ & Páncreas & $226.910(4,2 \%)$ & Páncreas & $205.332 \quad(4,9 \%)$ \\
\hline 8 & Próstata & 358.989 (3,8\%) & Leucemia & $179.518(3,3 \%)$ & Ovario & $184.799 \quad(4,4 \%)$ \\
\hline 9 & Cérvico uterino & 311.365 (3,3\%) & Vejiga & $148.270(2,8 \%)$ & Esófago & $151.395 \quad(3,6 \%)$ \\
\hline 10 & Leucemia & $3.009 .006(3,2 \%)$ & $\begin{array}{l}\text { Linfoma No } \\
\text { Hogdkin }\end{array}$ & $145.969(2,7 \%)$ & Leucemia & $129.488 \quad(3,1 \%)$ \\
\hline
\end{tabular}


Tabla 2. Ranking de cánceres con mayor incidencia a nivel mundial y según sexo, AIRC 2018

\begin{tabular}{|c|c|c|c|c|c|c|}
\hline \multirow[t]{2}{*}{ Ranking } & \multicolumn{2}{|c|}{ Total } & \multicolumn{2}{|c|}{ Hombres } & \multicolumn{2}{|c|}{ Mujeres } \\
\hline & $\begin{array}{l}\text { Tipo de } \\
\text { cáncer }\end{array}$ & $\begin{array}{c}\text { Casos nuevos } \\
\text { n (\%) }\end{array}$ & $\begin{array}{l}\text { Tipo de } \\
\text { cáncer }\end{array}$ & $\begin{array}{c}\text { Casos nuevos } \\
\text { n (\%) }\end{array}$ & $\begin{array}{l}\text { Tipo de } \\
\text { cáncer }\end{array}$ & $\begin{array}{c}\text { Casos nuevos } \\
\text { n (\%) }\end{array}$ \\
\hline 1 & Pulmón & $2.093 .876(11,6 \%)$ & Pulmón & $1.368 .524(14,5 \%)$ & Mama & $2.088 .849(24,2 \%)$ \\
\hline 2 & Mama & $2.088 .849(11,6 \%)$ & Próstata & $1.276 .106(13,5 \%)$ & Colorrectal & $823.303 \quad(9,5 \%)$ \\
\hline 3 & Colorrectal & $1.849 .518(10,2 \%)$ & Colorrectal & $1.026 .215(10,9 \%)$ & Pulmón & $725.352 \quad(8,4 \%)$ \\
\hline 4 & Próstata & $1.276 .106(7,1 \%)$ & Estómago & $683.754(7,9 \%)$ & Cérvico uterino & $569.847(6,6 \%)$ \\
\hline 5 & Estómago & $1.033 .701(5,7 \%)$ & Hígado & $596.574(6,3 \%)$ & Tiroides & $436.344 \quad(5,1 \%)$ \\
\hline 6 & Hígado & $841.080(4,7 \%)$ & Vejiga & $424.082(4,5 \%)$ & Corpus uterino & $382.069 \quad(4,4 \%)$ \\
\hline 7 & Esófago & $572.034(3,2 \%)$ & Esófago & $399.699(4,2 \%)$ & Estómago & $349.947 \quad(4,1 \%)$ \\
\hline 8 & Cérvico uterino & $569.847(3,2 \%)$ & $\begin{array}{l}\text { Linfoma } \\
\text { no Hodgkin }\end{array}$ & $284.713(3,0 \%)$ & Ovario & $295.414 \quad(3,4 \%)$ \\
\hline 9 & Tiroides & $567.233(3,1 \%)$ & Riñones & $254.507(2,7 \%)$ & Hígado & $244.506 \quad(2,8 \%)$ \\
\hline 10 & Vejiga & $549.393(3,0 \%)$ & Leucemia & $249.454(2,6 \%)$ & $\begin{array}{l}\text { Linfoma } \\
\text { no Hodgkin }\end{array}$ & $224.877 \quad(2,6 \%)$ \\
\hline
\end{tabular}

2016 (Figura 2). El Departamento de Estadística e Información en Salud (DEIS) señaló que, en el año 2016, el cáncer fue la segunda causa de muerte en Chile (Figura 1$)^{10}$, seguido por las enfermedades cardiovasculares (ECV). Sin embargo, en regiones como Arica y Parinacota, Tarapacá, Antofagasta, Maule, Biobío, Los Lagos y Aysén, la mortalidad por cáncer es la primera causa de muerte ${ }^{6}$.

$\mathrm{Al}$ revisar la mortalidad por cáncer en Chile, el cáncer de pulmón $(12,6 \%)$ se ubica en la posición uno del ranking de mortalidad, seguido del cáncer de estómago con $12,2 \%$, y cáncer colorrectal con $11,1 \%$. Al revisar estas cifras de mortalidad según sexo encontramos que el cáncer de estómago y próstata son responsables de más de $30 \%$ de las muertes en hombres. En el caso de las mujeres, la tendencia es similar a la mundial siendo el cáncer de mama, colorrectal y pulmón los tres cánceres con mayor nivel de mortalidad en mujeres chilenas $^{5}$ (Tabla 3 ).

Una reciente publicación destacó que, a pesar de que las ECV son las que más muertes provocan, es el cáncer la primera causa de muerte en países desarrollados. Este interesante trabajo fue realizado por PURE (Prospective Urban and Rural Epidemiologic), estudio que reúne información de 21 países, entre ellos Chile. Esta misma tendencia

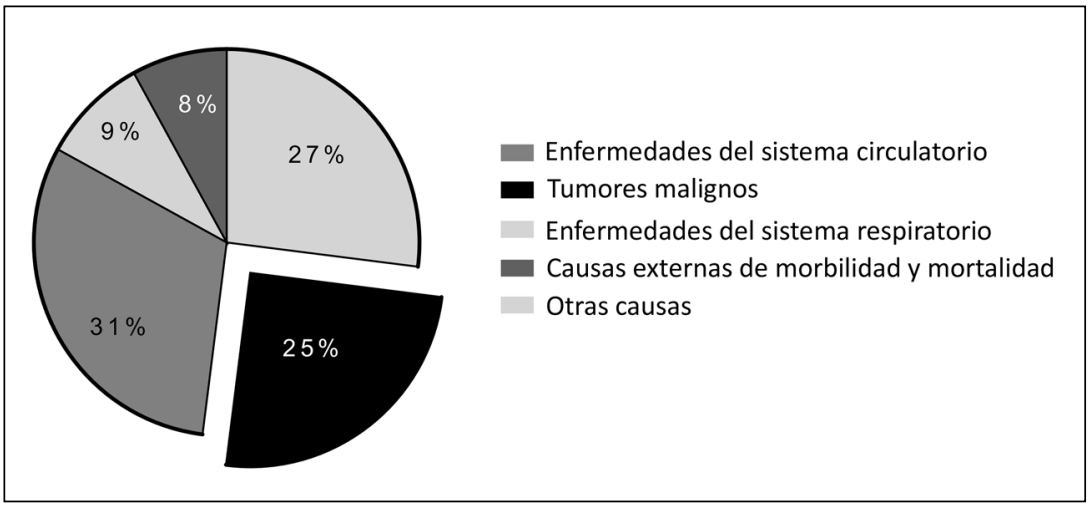

Figura 1. Principales causas de muertes en Chile, año 2016. 


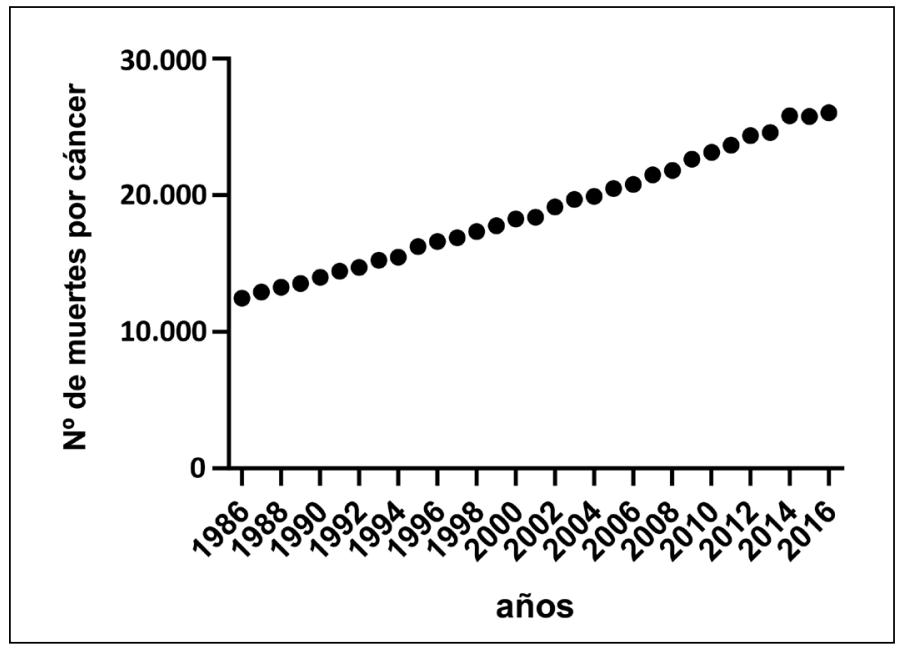

Figura 2. Número de muertes por cáncer en Chile (período año 1986 y 2016). Elaboración propia datos extraídos de Internacional Agency for Research on Cancer y DEIS6.

Tabla 3. Ranking de los cánceres con mayor nivel de mortalidad según sexo en Chile, AIRC 2018

\begin{tabular}{|c|c|c|c|c|c|c|}
\hline \multirow[t]{2}{*}{ Ranking } & \multicolumn{2}{|c|}{ Total } & \multicolumn{2}{|c|}{ Hombres } & \multicolumn{2}{|c|}{ Mujeres } \\
\hline & $\begin{array}{l}\text { Tipo de } \\
\text { cáncer }\end{array}$ & $\begin{array}{c}\text { Muertes } \\
\text { n (\%) }\end{array}$ & $\begin{array}{l}\text { Tipo de } \\
\text { cáncer }\end{array}$ & $\begin{array}{c}\text { Muertes } \\
\text { n (\%) }\end{array}$ & $\begin{array}{l}\text { Tipo de } \\
\text { cáncer }\end{array}$ & $\begin{array}{c}\text { Muertes } \\
\text { n (\%) }\end{array}$ \\
\hline 1 & Pulmón & $3.581(12,6 \%)$ & Estómago & $2.342(15,6 \%)$ & Mama & $1.688(12,5 \%)$ \\
\hline 2 & Estómago & $3.478(12,1 \%)$ & Próstata & $2.270(15,1 \%)$ & Colorrectal & $1.588(11,8 \%)$ \\
\hline 3 & Colorrectal & $3.144(11,1 \%)$ & Pulmón & $2.056(13,7 \%)$ & Pulmón & $1.525(11,3 \%)$ \\
\hline 4 & Próstata & $2.270 \quad(8,0 \%)$ & Colorrectal & $1.556(10,4 \%)$ & Estómago & $1.136 \quad(8,3 \%)$ \\
\hline 5 & Mama & $1.688 \quad(5,9 \%)$ & Hígado & $781 \quad(5,2 \%)$ & Vesícula & $1.063 \quad(7,9 \%)$ \\
\hline 6 & Vesícula & $1.623 \quad(5,7 \%)$ & Páncreas & $763 \quad(5,1 \%)$ & Páncreas & $816 \quad(6,1 \%)$ \\
\hline 7 & Páncreas & $1.579 \quad(5,6 \%)$ & Riñones & $637 \quad(4,3 \%)$ & Cérvico uterino & $725 \quad(5,4 \%)$ \\
\hline 8 & Hígado & $1.448 \quad(5,1 \%)$ & Vesícula & $560 \quad(3,7 \%)$ & Hígado & $667 \quad(5,0 \%)$ \\
\hline 9 & Riñones & $981 \quad(3,4 \%)$ & Leucemia & $448(3,0 \%)$ & Ovario & $470 \quad(3,5 \%)$ \\
\hline 10 & Leucemia & $883 \quad(3,1 \%)$ & $\begin{array}{l}\text { Linfoma de } \\
\text { no-Hodgkin }\end{array}$ & $423 \quad(2,8 \%)$ & Leucemia & $435 \quad(3,2 \%)$ \\
\hline
\end{tabular}

se ha observado en algunos países de mediano y alto ingreso económico como Chile, Argentina, Polonia y Turquía, el estudio muestra que en la actualidad existe una mayor proporción de muertes por cáncer en comparación a ECV en estos países. En contraste a las cifras de países como India y Pakistán (países de bajos ingresos), donde las muertes provocadas por las ECV son muy superiores a las causadas por cáncer ${ }^{11}$.

Según los datos de Globocan 2018, en Chile se diagnosticaron 53.365 nuevos casos de cáncer, li- derado por cáncer de próstata, colorrectal, mama, estómago, pulmón y vesícula. Al revisar por sexo, el cáncer de próstata, estómago y colorrectal lideran el ranking en los hombres, mientras que el ranking en mujeres está liderado por cáncer de mama, colorrectal y vesícula ${ }^{5,12}$ (Tabla 4 ).

El número de muertes atribuidas al cáncer en Chile se ha incrementado de $21 \%$ en 1990 a $26 \%$ en 2017; de hecho, 11 de cada 100 personas tienen un alto riesgo de mortalidad asociada a cáncer antes de los 75 años $^{13}$. 
Tabla 4. Ranking de los cánceres con mayor incidencia según sexo en Chile, AIRC 2018

\begin{tabular}{|clclllll|}
\hline Ranking & Tipo de & $\begin{array}{c}\text { Total } \\
\text { cáncer }\end{array}$ & $\begin{array}{c}\text { Muertes } \\
\mathbf{n}(\%)\end{array}$ & $\begin{array}{l}\text { Tipo de } \\
\text { cáncer }\end{array}$ & $\begin{array}{c}\text { Hombres } \\
\text { Casos nuevos } \\
\mathbf{n}(\%)\end{array}$ & $\begin{array}{l}\text { Tipo de } \\
\text { cáncer }\end{array}$ & $\begin{array}{c}\text { Mujeres } \\
\text { Casos nuevos } \\
\text { n (\%) }\end{array}$ \\
\hline 1 & Próstata & $6.576(12,3 \%)$ & Próstata & $6.576(23,9 \%)$ & Mama & $5.393(20,8 \%)$ \\
\hline 2 & Colorrectal & $5.914(11,1 \%)$ & Estómago & $3.429(12,5 \%)$ & Colorrectal & $2.912(11,3 \%)$ \\
\hline 3 & Mama & $5.393(10,1 \%)$ & Colorrectal & $3.002(10,9 \%)$ & Vesícula & $1.848 \quad(7,1 \%)$ \\
\hline 4 & Estómago & $5.162(9,7 \%)$ & Pulmón & $2.163 \quad(7,9 \%)$ & Estómago & $1.733 \quad(6,7 \%)$ \\
\hline 5 & Pulmón & $3.873(7,3 \%)$ & Riñón & $1.221 \quad(4,4 \%)$ & Pulmón & $1.710 \quad(6,6 \%)$ \\
\hline 6 & Vesícula & $2.699(5,1 \%)$ & Testículos & $981 \quad(3,6 \%)$ & Cérvico uterino & $1.549 \quad(6,0 \%)$ \\
\hline 7 & Riñón & $1.951 \quad(3,7 \%)$ & Vejiga & $964 \quad(3,5 \%)$ & Corpus uterino & $933 \quad(3,6 \%)$ \\
\hline 8 & Páncreas & $1.635(3,1 \%)$ & Hígado & $871 \quad(3,2 \%)$ & Tiroides & $901 \quad(3,5 \%)$ \\
\hline 9 & Hígado & $1.582 \quad(3,0 \%)$ & Vesícula & $851 \quad(3,1 \%)$ & Páncreas & $841 \quad(3,2 \%)$ \\
\hline 10 & Cérvico uterino & $1.549(2,9 \%)$ & Linfoma & $847 \quad(3,1 \%)$ & Ovario & $841 \quad(3,2 \%)$ \\
\hline
\end{tabular}

Proyecciones para el año 2040

Según las proyecciones de Cancer Research UK y AIRC, se pronostica que en el año 2040 se diagnosticarán más de 29,5 millones de nuevos casos de cáncer en el mundo, esto dado por las tendencias recientes en la incidencia de cánceres y el envejecimiento de la población a nivel mundial, el cual se caracteriza por un aumento de las hiperplasias ${ }^{14}$. Este aumento será equivalente a $62,9 \%$ en comparación a las cifras de cáncer en el 2018 (18,1 millones) y se espera que sea más alto en los hombres (aumento de 67,6\%) que en las mujeres (incremento de 55,3\%) ) $^{15,16}$.

Un estudio publicado en 2016, por Smittenaar y cols., muestra que los cánceres con mayor aumento a nivel mundial serán el cáncer de tiroides, hígado, oral y riñón, mientras que la mayor incidencia la presentarán el cáncer de mama en las mujeres y el cáncer de próstata para los hombres ${ }^{17}$.

Por otra parte, según las estimaciones realizadas por AIRC, en Chile para el año 2040, habrá 94.807 nuevos casos de cáncer, lo que corresponde a un aumento de 77,6\% en los próximos 21 años. En la actualidad, el cáncer ocasiona 28.443 muertes en Chile, las que llegarán a 55.698 para el año 2040 según las estimaciones de $\mathrm{AIRC}^{16,18}$.

\section{Medidas de control asociadas al futuro escenario del cáncer en Chile}

Las estadísticas presentadas en esta revisión entregan evidencia concreta de que el cáncer será uno de los desafíos sanitario de las próximas décadas, tanto en Chile como en el Mundo. Si bien estas proyecciones tienen el objetivo de informar los cambios epidemiológicos relacionados a la incidencia y mortalidad por cáncer en el mundo, también cumplen con el objetivo de orientar a los sistemas de salud los gobiernos para que puedan tomar medidas que ayuden a reducir el costo económico, social, personal y familiar asociado al cáncer. Este último objetivo es clave si consideramos que $15 \%$ de los países con ingreso medio y bajo en el mundo cuentan con servicios integrales para su tratamiento, frente a $90 \%$ de países de ingresos altos ${ }^{4}$.

Chile enfrentará un cambio en el ranking de las principales causas de muerte, estimándose que cáncer se posicionará en el primer lugar ${ }^{11}$. Por ende, dichas proyecciones son claves para que las entidades gubernamentales y sistemas de salud nacional implementen medidas de control necesarias, desde las dirigidas a la prevención hasta el diagnóstico, tratamiento, gestión, cuidados paliativos y vigilancia.

Es esencial que Chile aumente la inversión en prevención, sobre todo considerando que la baja proporción de la población chilena cumple con recomendaciones de alimentación saludable. Según datos de la Encuesta Nacional de Consumo Alimentario (ENCA), solo 52\% de los chilenos 
cumple con las recomendaciones de consumo de frutas y verduras, $25 \%$ con la recomendación de consumo de legumbres; no obstante, $44 \%$ de los individuos consume cereales procesados, $95 \%$ de la población consume carnes rojas y procesadas y más de $90 \%$ reporta consumir azúcares y bebidas azucaradas, llegando a un promedio de $20 \mathrm{~g} /$ día de azúcares ${ }^{19,20}$. La reducción de estas conductas de consumo alimentario no saludable es sumamente relevante en la prevención de cáncer, sobre todo si tomamos en cuenta que 913.090 muertes por cáncer, registradas en el 2018 fueron atribuidas a una alimentación no saludable ${ }^{21}$. Además, a este escenario se suma que más de $89 \%$ de los chilenos reportan ser físicamente inactivo en su tiempo libre, lo cual es un factor de riesgo para el desarrollo de cáncer considerando que $10 \%$ de las muertes por cáncer son atribuibles a ser físicamente inacti$\mathrm{vo}^{22}$. A este desalentador escenario se suma la alta prevalencia exceso de peso en Chile donde más de $80 \%$ de la población presenta mal nutrición por exceso, siendo uno de los principales factores de riesgo para cáncer. La agencia de WCRF estimó que el exceso de peso corporal y la obesidad se asocian a un mayor riesgo de desarrollar 12 tipos diferentes de cánceres, entre ellos cáncer de mama, próstata, colorrectal y vesícula biliar. Lo cual es interesante considerando que estos cuatro cánceres mencionados son los que presentan actualmente la mayor prevalencia en Chile ${ }^{23}$. Por lo cual, si queremos frenar las proyecciones en el aumento de cánceres en Chile es necesario implementar medidas concretas para su prevención.

Otro aspecto clave a considerar para cambiar el impacto que tendrá el cáncer en Chile es trabajar en la implementación de programas de detección temprana de cáncer ${ }^{24}$. Estos se basan en dos aspectos esenciales como lo es el aumento del número de screening realizados en la población, lo cual tendrá un impacto directo en la detección temprana de cáncer ${ }^{24}$. El poder detectar los cánceres en una etapa temprana tendría implicaciones importantes en aumentar la sobrevivencia de pacientes con cáncer, como también así reducir el costo asociado a la enfermedad para el paciente, familiares y el sistema de salud. Otro aspecto clave que se debería considerar en base a la proyección del escenario de cáncer en Chile es el tratamiento paliativo de personas con cáncer ${ }^{24}$. Se necesita una mayor inversión en programas que fortalezcan y mejoren el tratamiento paliativo de cáncer en
Chile y erradiquen las desigualdades en el acceso a tratamiento farmacológico, físico y psicológico de pacientes con cáncer.

\section{Conclusión}

En Chile la incidencia y mortalidad por cáncer sigue aumentando de manera exponencial a través de los años y se proyecta que se transformará en la primera causa de muerte en todas las regiones del país. Los cánceres más prevalentes corresponden al de próstata, mama, pulmón, colorrectal y vesícula biliar. Considerando que el $40 \%$ de los cánceres se relacionan con estilos de vida no saludables, trabajar en la prevención de los factores de riesgo modificables representa una oportunidad para el diseño de políticas públicas que permitan o fomenten cambios en los ambientes y conductas individuales de riesgo, favoreciendo una mejora en la salud de la población. Así mismo, se requiere mayor investigación que permita identificar y cuantificar la influencia de factores modificables para el desarrollo del cáncer.

\section{Referencias}

1. World Health Organization. Latest global cancer data: Cancer burden rises to 18.1 million new cases and 9.6 million cancer deaths in 2018 [Internet]. 2018. Available from: http://gco.iarc.fr/ [citado el 29 de julio de 2019].

2. Bray F, Jemal A, Grey N, Ferlay J, Forman D. Global cancer transitions according to the Human Development Index (2008-2030): a population-based study. Lancet Oncol [Internet]. 2012; 13 (8): 790-801. Available from: http://www.ncbi.nlm.nih.gov/pubmed/22658655 [citado el 5 de agosto de 2019].

3. The Economist. Control del cáncer, acceso y desigualdad en América Latina. Una historia de luces y sombras [Internet]. 2017. Available from: https://eiuperspectives. economist.com/sites/default/files/images/Cancer_control_access_and_inequality_in_Latin_America_SPANISH.pdf [citado el 5 de agosto de 2019].

4. Deutsche Welle (DW). OMS advierte de drástico aumento de casos de cáncer | El Mundo | DW | 04.02.2020 [Internet]. 2020. Available from: https://www.dw.com/ es/oms-advierte-de-drástico-aumento-de-casos-de-cáncer/a-52250903 [citado el 13 de mayo de 2019].

5. Global Cancer Observatory. New Global Cancer Data: GLOBOCAN 2018 | UICC. 2018. 
6. World Health Organization. GICR - The Global Initiative for Cancer Registry Development [Internet]. Available from: https://gicr.iarc.fr/ [citado el 5 de agosto de 2019].

7. International Agency for Research on Cancer. Global Cancer Observatory [Internet]. Available from: http:// gco.iarc.fr/ [citado el 21 de octubre de 2019].

8. Ferrini K. Lifestyle, nutrition and breast cancer: facts and presumptions for consideration. Ecancermedicalscience. 2015; 9.

9. Castelló A, Martín M, Ruiz A, Casas AM, Baena-Cañada JM, Lope V, et al. Lower Breast Cancer Risk among Women following the World Cancer Research Fund and American Institute for Cancer Research Lifestyle Recommendations: EpiGEICAM Case-Control Study. Sapino A, editor. PLoS One. 2015; 10 (5): e0126096.

10. Departamento de estadísticas e informaciones de salud. Estadísticas de Natalidad y Mortalidad - DEIS [Internet]. Available from: http://www.deis.cl/estadisticas-de-natalidad-y-mortalidad/ [citado el 12 de agosto de 2019].

11. Yusuf S, Joseph P, Rangarajan S, Islam S, Mente A, Hystad P, et al. Modifiable risk factors, cardiovascular disease, and mortality in 155,722 individuals from 21 high-income, middle-income, and low-income countries (PURE): a prospective cohort study. Lancet (London, England) [Internet]. 2019; 0 (0). Available from: http:// www.ncbi.nlm.nih.gov/pubmed/31492503 [citado el 7 de octubre de 2019].

12. GLOBOCAN. GLOBOCAN 2012: Estimated Cancer Incidence, Mortality and Prevalence Worldwide in 2012 v1.0 [Internet]. Available from: https:// publications.iarc.fr/Databases/Iarc-Cancerbases/ GLOBOCAN-2012-Estimated-Cancer-Incidence-Mortality-And-Prevalence-Worldwide-In-2012-V1.0-2012 [citado el 12 de agosto de 2019].

13. Kielstra P. Control del cáncer, acceso y desigualdad en américa latina: Una historia de luces y sombras. Econ
Intell Unit 2017; 74.

14. Campisi J. Aging, cellular senescence, and cancer. Annu Rev Physiol 2013; 75: 685-705.

15. Cancer Research UK. Worldwide cancer incidence statistics | Cancer Research UK. 2018.

16. International Agency for Reseach on Cancer. Cancer Tomorrow. 2018.

17. Smittenaar CR, Petersen KA, Stewart K, Moitt N. Cancer incidence and mortality projections in the UK until 2035. Br J Cancer 2016; 115 (9): 1147-55.

18. International Agency for Research on Cancer. Global Cancer Observatory.

19. Ministerio de Salud de Chile. Encuesta Nacional de Consumo Alimentario [Internet]. 2014. Available from: https://www.minsal.cl/sites/default/files/ENCA_FINAL_DIC_2014.pdf [citado el 9 de diciembre de 2018].

20. Ministerio de Salud de Chile. Encuesta Nacional de Salud 2016-2017 [Internet]. 2018. Available from: http:// web.minsal.cl/wp-content/uploads/2017/11/ENS-201617_PRIMEROS-RESULTADOS.pdf [citado el 27 de febrero de 2018].

21. GBD 2017 Diet Collaborators A, Sur PJ, Fay KA, Cornaby L, Ferrara G, Salama JS, et al. Health effects of dietary risks in 195 countries, 1990-2017: a systematic analysis for the Global Burden of Disease Study 2017. Lancet (London, England) [Internet] 2019; 393 (10184): 1958-72. Available from: http://www.ncbi. nlm.nih.gov/pubmed/30954305 [citado el 7 de octubre de 2019].

22. Lee I-M, Shiroma EJ, Lobelo F, Puska P, Blair SN, Katzmarzyk PT. Impact of Physical Inactivity on the World's Major Non-Communicable Diseases. Lancet 2012.

23. Parra-Soto S, Martínez-Sanguinetti MA, Petermann-Rocha F, Celis-Morales C. Obesity and Cancer - The two scenarios that Chile will lead. Rev Med Chil. 2019.

24. WHO. Cancer [Internet]. 2020. Available from: https:// www.who.int/news-room/fact-sheets/detail/cancer [citado el 13 de mayo de 2019]. 